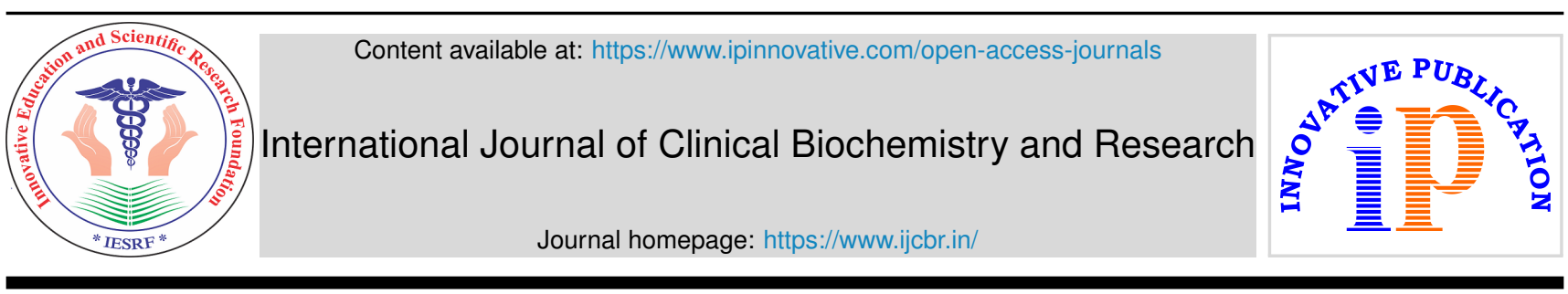

\title{
Editorial
}

\section{Effectiveness of CRISPR based tools as alternative methods for diagnosing COVID-19}

\author{
Shefali Mehta ${ }^{1, *}$, Aditi Mehta ${ }^{2}$ \\ ${ }^{1}$ Dept. of Biochemistry, RNT Medial College, Udaipur, Rajasthan, India \\ ${ }^{2}$ Pacific Institute of Medical Science, Udaipur, Rajasthan, India
}

\section{A R T I C L E I N F O}

\section{Article history:}

Received 27-09-2021

Accepted 29-09-2021

Available online 08-10-2021

\begin{abstract}
A B S T R A C T
The Clustered Regularly Interspaced Short Palindromic Repeats (CRISPR)-Cas (CRISPR - Associated Proteins) systems are prokaryotic adaptive immune mechanisms that are used for cleaving the invading nucleic acids in nature. Due to this reason, the CRISPR-based tools are used in numerous applications like genome and transcriptome engineering, gene therapy, epigenome editing and many others. In addition, the issue of errors in these tests is also increasing at a very rapid pace, due to which their reliability and efficiency and effectiveness are getting severely hampered. Therefore, it may not be wrong to say that there is a still a lot more room for improvement and development to enable, widespread, rapid, and scalable testing of the COVID-19 virus. In this regard, the RT-qPCR tests can be used as a routine molecular diagnostic method for detecting any COVID-19 symptoms. However, there are various limitations that mar the use and implementation of the RT-qPCR tests. But a few of these shortcomings can be overcome by using alternative molecular diagnostic methods like the CRISPR based tests.
\end{abstract}

This is an Open Access (OA) journal, and articles are distributed under the terms of the Creative Commons Attribution-NonCommercial-ShareAlike 4.0 License, which allows others to remix, tweak, and build upon the work non-commercially, as long as appropriate credit is given and the new creations are licensed under the identical terms.

For reprints contact: reprint@ipinnovative.com

\section{Introduction}

Cases of pneumonia of unknown origin began to be reported and recorded in Chinese province of Wuhan, since the month of December in the year 2019. ${ }^{1}$ Through extensive research studies and examinations, it was found that a new type of coronavirus was causing these cases. COVID19 , which is a result of SARS-CoV-2, began spreading to many other countries in a very short period and soon it became a concern for global health. According to several studies, one very effective way of combating and managing COVID-19 is by detecting infected individuals as early as possible, along with isolating such people and putting them in quarantine.

However, it has been observed that many people do not show the symptoms of being infected with SARS-CoV-2

\footnotetext{
* Corresponding author.

E-mail address: shefalimehta202@gmail.com (S. Mehta).
}

and therefore they do not also show any signs or symptoms of COVOD-19 during their diagnosis. ${ }^{2}$ Such people are also termed as asymptomatic or pre-symptomatic. These individuals can also transmit the virus to other healthy individuals, acting as a silent carrier. On this basis, it can be said that carrying out widespread testing is very important, even if there are no absolute or dependable clinical findings. Such testing should start with individuals who are under suspicion of being infected with the virus. ${ }^{3}$

One of the ways to detect SARS-CoV-2 infection is by checking for the presence of viral antigens or viral RNA in the respiratory samples. Presence of antibodies against the viral proteins in the blood sample can also be a way to detect for the SARS-CoV-2 virus among individuals. Recently, Rapid Antigen Tests (RATs) also have been developed to quicken the process of testing and diagnosis for the COVID19 virus. ${ }^{4}$ However, it should be kept in mind that the sensitivity for such tests is very low, which reduces their 
overall reliability and accuracy.

It takes only a few days for the antibodies of SARSCoV-2 to reach detectable levels in the blood. In only a few days to a week, the antibodies can be absorbed in the blood after first observation of COVID-19 symptoms. ${ }^{5}$ Due to this very reason, adequate levels of sensitivity for diagnosing acute infection of the virus cannot be detected by using the RATs and other such tests. In contrast to it, the use of nucleic acid tests can detect even the very small quantities of viruses in the clinical samples. This is one of the reasons for the development of RT-qPCR tests. Further, their use for detecting the SARS-CoV-2 throughout the world is also increasing at a fast pace. ${ }^{6}$

Even though the use of RT-qPCR has played a pivotal role in combating the COVID-19 virus, there are a still of developments and updates that need to be carried out before it is used in the desired manner. Furthermore, these tests require various types of equipment and tools, in addition to highly skilled and trained manpower or personnel to use them. ${ }^{7}$ It is because of this reason alone; the use of RT-qPCR tests is limited to only a few centralised laboratories. In addition, the issue of errors in these tests is also increasing at a very rapid pace, due to which their reliability and efficiency and effectiveness are getting severely hampered. Therefore, it may not be wrong to say that there is a still a lot more room for improvement and development to enable, widespread, rapid, and scalable testing of the COVID-19 virus. ${ }^{8}$

The Clustered Regularly Interspaced Short Palindromic Repeats (CRISPR)-Cas (CRISPR - Associated Proteins) systems are prokaryotic adaptive immune mechanisms that are used for cleaving the invading nucleic acids in nature. Due to this reason, the CRISPR-based tools are used in numerous applications like genome and transcriptome engineering, gene therapy, epigenome editing and many others. ${ }^{9}$ Today, an increasing number of studies are using the CRISPR-Case systems for detecting nucleic acid and pathogen diagnosis. One of the key benefits of using them is that they are fast, cost effective, easy to use and portable, apart from being sensitive and specific. Moreover, no complicated tools are required for using this method. ${ }^{10}$

\section{Standard Methods for Molecular Detection of SARS-CoV-2}

One of the most effective ways of detecting SARS-CoV-2 throughout the world is through the RT-qPCR tests. Once the RNA has been extracted from the clinical samples, the viral RNA is converted into complementary DNA (cDNA) by using the reverse transcription (RT) in this technique. ${ }^{4}$ The cDNA is then amplified using the polymerase chain reaction (PCR). The PCR can be used for detecting realtime amplification of the target nucleic acids. This is done by using the sequence-specific fluorescent-labelled oligonucleotides.

\section{Sensitivity and Specificity}

CRISPR based diagnostic tests have been observed to have high clinical sensitivity and specificity. ${ }^{2}$ Over the years various strategies have been used to enhance sensitivity and specificity of CRISPR based processes for detecting the SARS-CoV-2 virus. During the current study, it was found that using two different crRNAs can help in increasing the sensitivity and make the tests more resistant to any potential viral RNA mutations.

\section{Conclusion}

During the current study, it was observed that one very effective way of combating the SARS-CoV-2 virus is to increase the number of tests, along with making the tests feasible and available in numerous locations. In this regard, the RT-qPCR tests can be used as a routine molecular diagnostic method for detecting any COVID-19 symptoms. However, there are various limitations that mar the use and implementation of the RT-qPCR tests. But a few of these shortcomings can be overcome by using alternative molecular diagnostic methods like the CRISPR based tests.

\section{Conflict of Interest}

None.

\section{References}

1. Kaur M, Tiwari S. Protein based biomarkers for non-invasive Covid-19 detection. Sensing Bio-Sensing Res. 2020;29:100362. do1:10.1016/j.sbsr.2020.100362.

2. Clark T, Brendish N. Diagnostic accuracy of the FebriDx host response point-of-care test in patients hospitalised with suspected COVID-19. J Infect. 2020;81(4):607-13.

3. Mahmud T. CovXNet: A multi-dilation convolutional neural network for automatic COVID-19 and other pneumonia detection from chest X-ray images with transferable multi-receptive feature optimization. Comput Biol Med. 2020;122:103869. doi:10.1016/].compbiomed.2020.103869.

4. Tajima Y, Suda Y. A case report of SARS-CoV-2 confirmed in saliva specimens up to 37 days after onset: Proposal of saliva specimens for COVID-19 diagnosis and virus monitoring. J Infect Chemother. 2020;26(10):1086-9.

5. Yong SE. 2020.

6. Axell-House D. The estimation of diagnostic accuracy of tests for COVID-19: A scoping review. Journal of Infection. 2020;.

7. Böger B. Systematic review with meta-analysis of the accuracy of diagnostic tests for COVID-19. American journal of infection control. 2021;49(1):21-29.

8. Ong D, Claas E. Comparison of the GeneFinderTM COVID-19 Plus RealAmp Kit on the sample-to-result Platform ELITe InGenius to the national reference method: An added value of $\mathrm{N}$ gene target detection. Journal of Clinical Virology. 2020;132:104632-104632.

9. Khan AI, Shah JL, Bhat MM. CoroNet: A deep neural network for detection and diagnosis of COVID-19 from chest $\mathrm{x}$ ray images. Comput Methods Programs Biomed. 2020;196:105581. do1:10.1016/].cmpb.2020.105581.

10. Whitelaw S. Applications of digital technology in COVID19 pandemic planning and response. Lancet Digit Health. 2020;2(8):e435-40. do1:10.1016/S2589-7500(20)30142-4. 


\section{Author biography}

Shefali Mehta, Assistant Professor

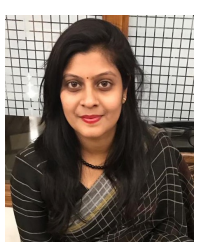

Aditi Mehta, Senior Resident

Cite this article: Mehta S, Mehta A. Effectiveness of CRISPR based tools as alternative methods for diagnosing COVID-19. Int J Clin Biochem Res 2021;8(3):150-152. 